\title{
Carbon ions and $\mathrm{X}$-rays induce pro-inflammatory effects in 3D oral mucosa models with and without PBMCs
}

\author{
VIKTORIA TSCHACHOJAN ${ }^{1}$, HENRIKE SCHROER ${ }^{1}$, NICOLE AVERBECK ${ }^{2}$ and WOLFGANG MUELLER-KLIESER ${ }^{1}$ \\ ${ }^{1}$ Institute of Pathophysiology, University Medical Center of the Johannes Gutenberg University Mainz, \\ Mainz; ${ }^{2}$ GSI Helmholtz Center for Heavy Ion Research, Darmstadt, Germany
}

Received June 24, 2014; Accepted July 30, 2014

DOI: 10.3892/or.2014.3441

\begin{abstract}
Oral mucositis is a severe complication of radiotherapy. Hence, it may constitute a serious medical safety risk for astronauts during extended space flights, such as missions to Mars, during which they are exposed to heavy-ion irradiation. For risk assessment of developing radiation-induced mucositis, a three-dimensional (3D) organotypic oral mucosa model was irradiated with ${ }^{12} \mathrm{C}$ heavy ions or X-rays. The present study focused mainly on early radiation-induced effects, such as the activation of nuclear factor $\kappa \mathrm{B}(\mathrm{NF} \kappa \mathrm{B})$ and the expression or release of pro-inflammatory marker molecules. The $3 \mathrm{D}$ oral mucosa models with or without peripheral blood mononuclear cells (PBMCs) were irradiated with X-rays or ${ }^{12} \mathrm{C}$ heavy ions followed by snap freezing. Subsequently, cryosections were derived from the specimens, which were immunostained for analysis of compactness, DNA double strand breaks (DSB) and activation of $\mathrm{NF \kappa B}$. Radiation-induced release of interleukin 6 (IL6) and interleukin 8 (IL8) was quantified by ELISA. Quantification of the DNA damage in irradiated mucosa models revealed distinctly more DSB after heavyion irradiation compared to X-rays at definite time points, suggesting a higher gene toxicity of heavy ions. NFKB activation was observed after treatment with $\mathrm{X}$-rays or ${ }^{12} \mathrm{C}$ particles. ELISA analyses showed significantly higher IL6 and IL8 levels after irradiation with X-rays and ${ }^{12} \mathrm{C}$ particles compared to non-irradiated controls, whereas co-cultures including PBMCs released 2 to 3 -fold higher interleukin concentrations compared to mucosa models without PBMCs. In this study, we demonstrated that several pro-inflammatory markers are induced by X-rays and heavy-ion irradiation within an oral mucosa model. This suggests that oral mucositis indeed poses a risk for astronauts on extended space flights.
\end{abstract}

Correspondence to: Dr Viktoria Tschachojan, Institute of Pathophysiology, University Medical Center of the Johannes Gutenberg University Mainz, Duesbergweg 6, D-55128 Mainz, Germany

E-mail: tschacho@uni-mainz.de

Key words: oral mucositis, irradiation, heavy ion, X-ray, oral mucosa model, PBMCs

\section{Introduction}

A manned mission to Mars constitutes a great vision of mankind, and is supported by several space agencies worldwide. However, the era of manned missions beyond the low Earth orbit from 1969 through 1972 ended over 40 years ago. Reasons for the cessation are, among others, various uncertainties regarding physiological effects of space radiation, isolation and microgravity during prolonged space flights of approximately 3 years. Space radiation consists of protons (87\%), $\alpha$-particles (12\%) and heavy ions (1\%) in solar particle events (SPEs) and galactic cosmic rays (GCRs). The dose deposition of heavy-ion radiation in SPEs and GCRs occurs inhomogeneously as the ions release their energy localized at a very high density along their trajectory. This distinguishes them from X-rays, which show a homogeneous dose deposition. Furthermore, these radiation types differ in their depth-dose profile. In contrast to X-rays, heavy-ion radiation is characterized by an inverted dose profile, i.e. the dose increases with the depth of penetration (1). Particularly highly ionizing heavy ions of GCRs can be hardly shielded exposing the crew members to a serious medical safety risk $(2,3)$, since the probability to be hit by a heavy ion beam increases with prolonged stay in space. Previous studies indicated that the risk of cancer-related mortality for extended exploration missions to the Moon and Mars varies from 0.2 to $15 \%(4,5)$, which is a very wide range. The uncertainties warrant further experimental analysis of the risk of radiation-induced diseases and complications during extended space flights. From experience in cancer therapy, we know that inflammation of the mucogingival membrane (oral mucositis) is a frequent complication of heavy-ion and X-ray radiotherapy (6-11). Patients often complain about burning and intolerance of spicy foods, xerostomia, bacterial, fungal or viral infections, dental caries and loss of taste (12). Furthermore, there is significant pain, odynodysphagia, subsequent dehydration, malnutrition, anorexia, cachexia, neurocognitive alterations and depression (13). Due to restricted possibilities of medical treatment in a space shuttle during prolonged missions, acute oral mucositis will most likely exacerbate to chronic mucositis with an increased probability of carcinogenesis (14-17).

It is known that in tight cell clusters the cells communicate with each other via molecular signaling (18-21). Since three-dimensional (3D) cultures mimic the in vivo situation 
much closer than conventional monolayer cultures, we chose this system as an adequate in vitro model for analyses of radiation-induced pro-inflammatory effects in early stages of oral mucositis. In the present study, we utilized a 3D mucosa model consisting of human keratinocytes and fibroblasts, where the keratinocytes interact with the fibroblasts in the layer below. In vivo, peripheral blood mononuclear cells (PBMCs) are indispensable for induction and progress of inflammatory processes. On that account, we additionally established co-cultures containing PBMCs to mimic the in vivo situation much closer, and compared the results to the cultures without immune cells.

The focus of the present study was on immediate and early pro-inflammatory effects after irradiation, where nuclear factor $\kappa \mathrm{B}(\mathrm{NF} \kappa \mathrm{B})$ activation and increased expression of the cytokines and chemokines are precursors of oral mucositis (22). By way of comparison, we additionally exposed the 3D mucosa model containing PBMCs to X-rays. Following irradiation with $\mathrm{X}$-rays or heavy ions $\left({ }^{12} \mathrm{C}\right)$, we analyzed the radiation impact on epithelium compactness, DNA damage, activation of NFKB and the release of the cytokine interleukin 6 (IL6) and chemokine IL8.

\section{Materials and methods}

Cell culture. Immortalized human gingival keratinocytes (IHGK; kindly provided by Professor Dr Tomakidi, Department of Oral Biotechnology, University Medical Center, Freiburg, Germany) were cultured in complete keratinocyte growth medium (PromoCell, Heidelberg, Germany). Immortalized human dermal fibroblasts HH4ded, kindly provided by Professor Dr Rodemann (Department of Radiation Oncology, University Hospital and Faculty of Medicine, Tuebingen, Germany) were cultured in Dulbecco's modified Eagle's medium (DMEM; high glucose with L-glutamine; Gibco $^{\circledR}$, Life Technologies GmbH, Darmstadt, Germany) supplemented with $10 \%$ (v/v) fetal bovine serum Gold (PAA Laboratories $\mathrm{GmbH}$, Coelbe, Germany). Cells were cultured under standard tissue culture conditions and tested routinely for mycoplasma contaminations (Venor ${ }^{\circledR}$ GeM Mycoplasma Detection kit; Minerva Biolabs, Berlin, Germany). PBMCs were isolated from buffy coats from healthy donors (Blood Bank University Medical Center Mainz, Germany). Initially, the buffy coat was diluted in sterile phosphate-buffered saline (PBS; 1:4). For isolation, separation medium LSM1077 (PAA Laboratories $\mathrm{GmbH}$ ) was overlayed with $25 \mathrm{ml}$ blood suspension. After a $20 \mathrm{~min}$ centrifugation $(1,200 \mathrm{x}$ g; Megafuge 1.0; Heraeus, Hanau, Germany) with breaks turned-off, the interphase was transferred to a new reaction tube, washed twice with PBS, and centrifuged for $10 \mathrm{~min}$ with $300 \mathrm{x}$ g. Numbers of viable PBMCs were determined upon microscopic observation in a Neubauer chamber after staining with $0.4 \%$ trypan blue.

Preparation of organotypic 3D oral mucosa model. Organotypic co-cultures were grown in gelatine-coated ThinCerts ${ }^{\mathrm{TM}}$ tissue culture inserts in deep 12-well plates (Greiner Bio-One $\mathrm{GmbH}$, Frickenhausen, Germany). The inserts consist of a polyester capillary pore membrane with $0.4-\mu \mathrm{m}$ pore size and a growth area of $1.131 \mathrm{~cm}^{2}$. Before initiating the organotypic cultures, ThinCerts ${ }^{\mathrm{TM}}$ were coated with $0.2 \%$ gelatin solution and incubated for $30 \mathrm{~min}$ at $37^{\circ} \mathrm{C}$. Subsequently, the fibroblasts were seeded in the 12 -well plates with $4 \times 10^{4}$ cells/insert. After 3 days, the inserts were transferred into deep well plates with $4.5 \mathrm{ml}$ of medium in each well and $3 \times 10^{5}$ cells/insert keratinocytes were added onto the fibroblast layer. After a further 3 days, the models were exposed to an air-liquid interface and incubated for another 20 days with media changes twice a week. Co-cultures with immune cells were generated by seeding $10^{6} \mathrm{PBMCs} /$ ThinCert ${ }^{\mathrm{TM}} 24 \mathrm{~h}$ before irradiation.

Irradiation. Irradiation was performed at the GSI Helmholtz Center for Heavy Ion Research in Darmstadt. Deep well plates with inserts containing organotypic cultures were completely filled with medium, sealed and transferred to the GSI. Due to a few uncertainties regarding cumulative dose for the Mars mission crew, we selected different doses for the irradiation. Control samples were left untreated (0 Gy), other samples were irradiated with 2 or $4 \mathrm{~Gy}$ of ${ }^{12} \mathrm{C}$ particle radiation with $\mathrm{E}_{\max } 155.26 \mathrm{MeV} / \mathrm{u}$, an LET of $300 \mathrm{keV} / \mu \mathrm{m}$ and a dose-rate of $\sim 0.6 \mathrm{~Gy} / \mathrm{min}$. To provoke a reliable radiation response in the mucosa model, we additionally irradiated with $10 \mathrm{~Gy}{ }^{12} \mathrm{C}$ ions in one experiment. For comparison, additional 3D cultures were irradiated with equivalent physical doses of X-rays $(250 \mathrm{kV}$, Isovolt DS1; Seifert, Germany; dose-rate $1 \mathrm{~Gy} / \mathrm{min})$. After irradiation, samples were transferred to new deep 12-well plates that were completely filled with medium and transported back to the Mainz laboratory, where the cultures were snap frozen 4, 8, 24 and $48 \mathrm{~h}$ after irradiation. In the course of transit and radiation, samples were kept at room temperature.

Immunohistochemistry and morphometric characterization of $3 D$ cultures. For histological and immunofluorescence stainings, snap frozen samples were embedded in Tissue-Tek ${ }^{\circledR}$ O.C.T. Compound (Sakura, Torrance, CA, USA) and cryosectioned $(10 \mu \mathrm{m})$ with a microtome (SLEE, Mainz, Germany). After overnight drying at room temperature, the sections were stored at $-20^{\circ} \mathrm{C}$. Hematoxylin (Carl Roth $\mathrm{GmbH} \& \mathrm{Co}$. KG, Karlsruhe, Germany) and eosin (HE) (Merck KGaA, Darmstadt, Germany) stainings were used for analyses of structural changes in irradiated tissue. For discrimination of epithelium and connective tissue as well as DNA damage and NFkB p50, immunofluorescence stainings were carried out. Sections were initially blocked with $2 \%$ donkey serum, following incubation with primary antibody for $1 \mathrm{~h}$ and 3 washing steps with PBS. Subsequently, samples were incubated with secondary antibody for $45 \mathrm{~min}$, washed again 4 times and mounted in ImmunoSelect Antifading Mounting Medium (Dianova GmbH, Hamburg, Germany) containing 4',6-diamidino-2-phenylindole (DAPI). For anti-phospho-histone $\mathrm{H} 2 \mathrm{AX}(\gamma \mathrm{H} 2 \mathrm{AX})$ staining, a preceding permeabilization of the cell membranes with a permeabilizing buffer [PBS $+0.5 \%(\mathrm{v} / \mathrm{v})$ Triton-X 100] was required. The following antibodies were used: monoclonal mouse anti-panCK (Dako, Hamburg, Germany), polyclonal rabbit anti NFאB p50 (Santa Cruz Biotechnology, Inc., Heidelberg, Germany), monoclonal mouse anti-phosphohistone H2AX Ser139 (Millipore Corporation, Billerica, MA, USA). Secondary detection was carried out with polyclonal donkey anti-rabbit DyLight 594 and polyclonal donkey antimouse DyLight 488 antibodies (Dianova $\mathrm{GmbH}$ ). Secondary 

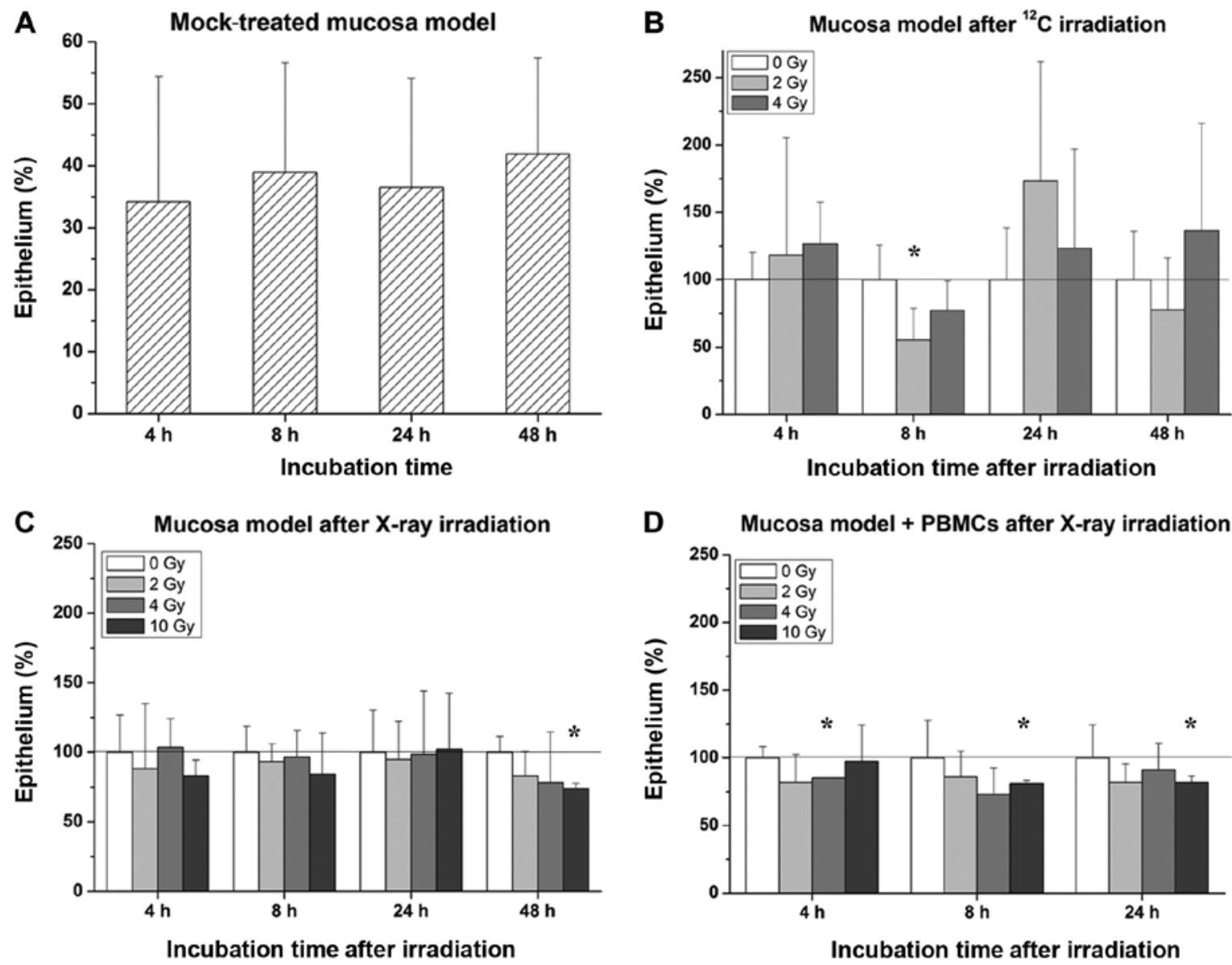

Figure 1. Morphometric evaluation of the proportion of epithelium with regard to the complete untreated and irradiated oral mucosa model. (A) In our mucosa model, the epithelial component comprised 30-40\%. Heavy ion radiation did not affect this proportion (B), while exposure to X-rays at a dose of $10 \mathrm{~Gy}$ caused shrinking of epithelium in cultures without immune cells after $48 \mathrm{~h}$ and in co-cultures containing immune cells after 4,8 and $24 \mathrm{~h}$, respectively (C and D). The evaluation was carried out from at least 3 images/culture and 3 independent experiments. Data are shown as means \pm standard deviations. Data were normalized to untreated controls, which were set to $100 \%$. ${ }^{*} \leq 0.05$ compared to untreated controls.

antibodies alone were used as negative controls. Sections were registered photographically using a BZ-8000 fluorescence microscope (Keyence Deutschland GmbH, Neu-Isenburg, Germany) for HE stainings and a confocal laser scanning microscope (Zeiss) was used for other stainings. For computer based analyses of epithelial proportion, the binary images of panCK stained cultures were quantified by Image J 1.46 using the plugin 'Live Histogram'. The values obtained were divided by the values obtained of binary images of DAPI-stained cultures, which were quantified in the same way. Compactness of oral mucosa models based on cell density and integrity was quantified from binary HE-stained cultures using the plugin 'Live Histogram' and related to the total area of each sample.

Quantification of DNA damage and NFKB activation. Computer-based analyses were carried out to quantify DNA

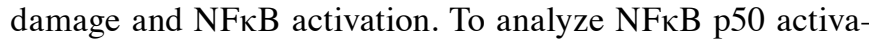
tion, only nuclear localized staining was quantified. For that purpose, the binary image of DAPI-stained cell nuclei served as template for $\mathrm{NF} \kappa \mathrm{B}$ p50 images. The intensity of the $\mathrm{NF \kappa B}$ p50 stainings in nuclei was quantified by ImageJ 1.46 using the plugin 'Measure RGB' (author, Wayne Rasband; version 1). The values obtained were divided by the values of binary nuclei stainings and normalized to untreated samples. For the evaluation of radiation-induced DNA double strand breaks (DSB), the foci number/nucleus of the DSB marker $\gamma \mathrm{H} 2 \mathrm{AX}$ was quantified. In order to do this, the 16 bit greyscale images obtained from $\gamma \mathrm{H} 2 \mathrm{AX}$ staining were counted by ImageJ using the plugin 'FociPicker3D 3D and 2D particle counter' (author, Guanghua Du; 16.04.2010, version 1). Subsequently, the foci number was divided by the corresponding visually determined cell number.

Cytokine quantification. Cell-free media supernatants of organotypic (co-) cultures were collected at the time of cryopreservation. Remaining solid particles were sedimented by centrifugation for $10 \mathrm{~min}$ with $400 \mathrm{x}$ g at $4^{\circ} \mathrm{C}$. The supernatants were stored at $-80^{\circ} \mathrm{C}$ until the samples were analyzed according to the manufacturer's instructions with human IL6 and IL8 ELISA MAX ${ }^{\mathrm{TM}}$ Standard Sets (BioLegend GmbH, Fell, Germany), respectively.

Statistical analysis. Data are shown as average values \pm standard error of the mean (SEM) from at least 3 independent experiments. Only the data regarding the epithelial component relative to respective grown culture are shown as the means \pm standard deviation. Statistical significance $(\mathrm{p} \leq 0.05)$ was determined using a two-tailed, unpaired Student's t-test. 
A HE stainings of mucosa model

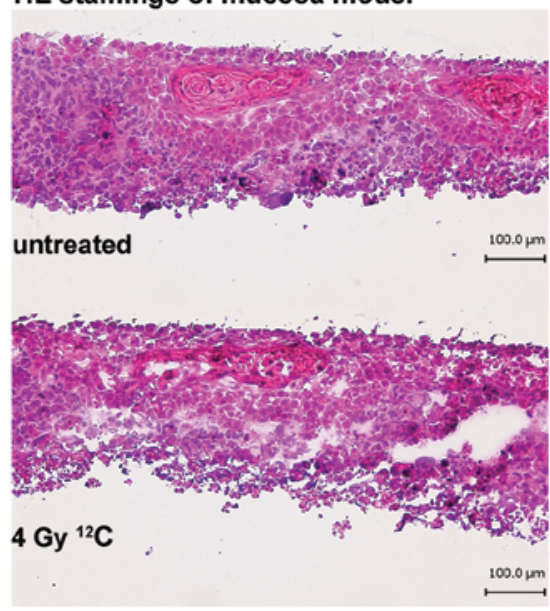

C

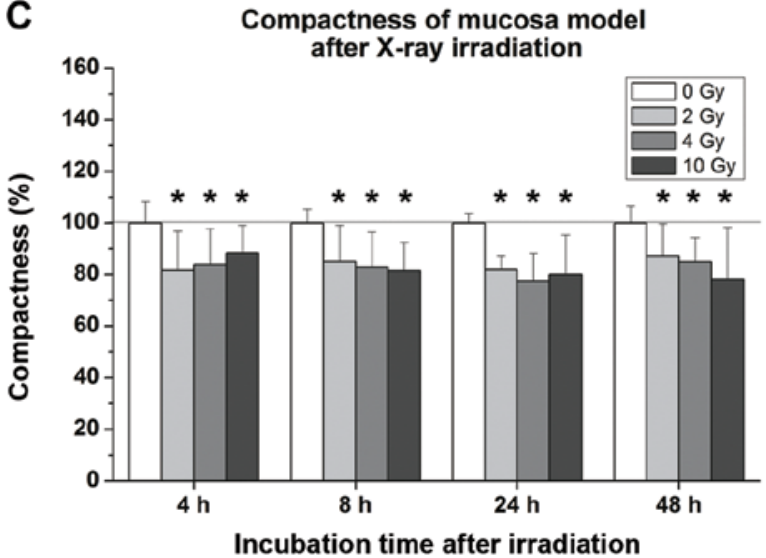

B
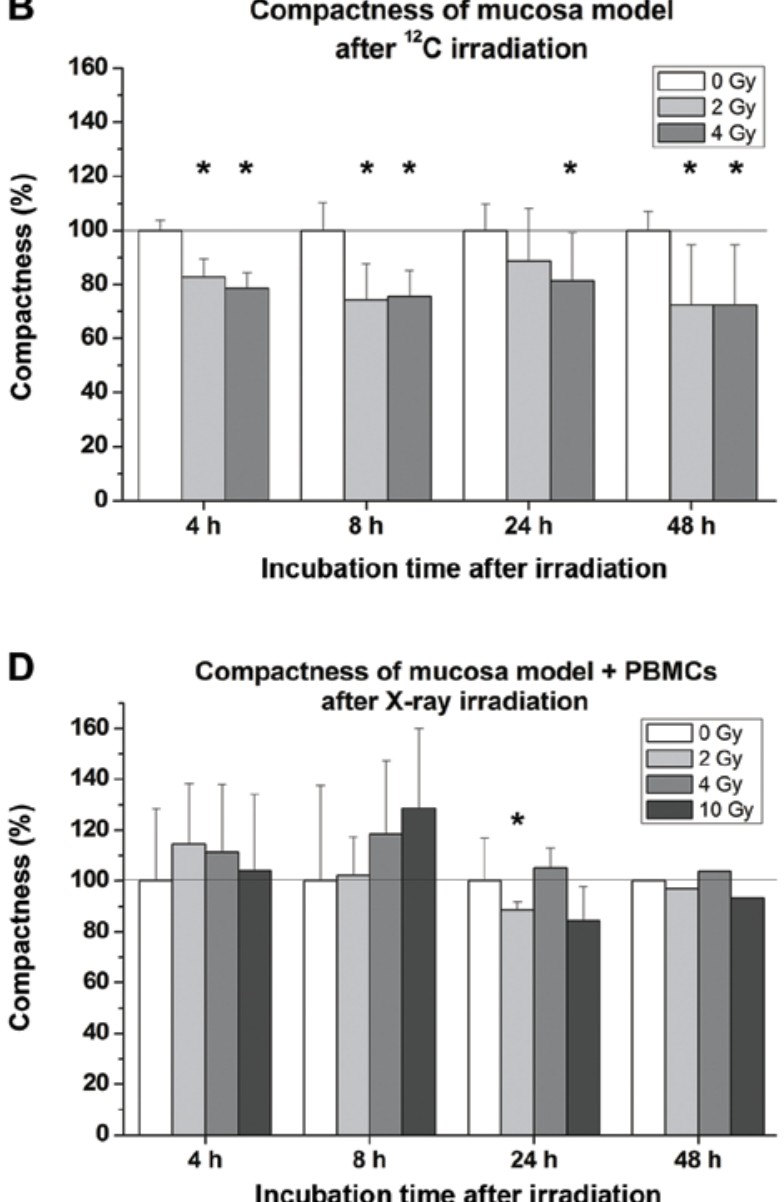

Figure 2. Radiation impact on compactness evaluated by computer-based analyses of HE stainings (A) after irradiation with ${ }^{12} \mathrm{C}$ particle beam (B) or $\mathrm{X}$-rays (C and D). Data were normalized to untreated controls, which were set to $100 \%$. Values are shown as means $\pm \mathrm{SEM}$ ( $\mathrm{n}=3$ ). ${ }^{*} \mathrm{p} \leq 0.05$ compared to untreated controls.

Statistically significant alterations marked with an asterisk $\left(^{*}\right)$ refer to the untreated control samples and those with a hash $\left(^{\#}\right)$, refer to the corresponding dose at $4 \mathrm{~h}$.

\section{Results}

Radiation impact on epithelium. The epithelial proportion of oral mucosa models was quantified from panCK stained cultures using at least 3 images of the same 3D culture. In our mucosa model, the epithelial component comprised $\sim 30-40 \%$ (Fig. 1A). Heavy-ion irradiation within a dose range of up to $4 \mathrm{~Gy}$ showed no systematic effect on the proportion of the epithelium, as depicted in Fig. 1B, whereas exposure to X-rays at a dose of 10 Gy caused a shrinkage of the epithelium in cultures without immune cells after $48 \mathrm{~h}$ and in co-cultures containing immune cells after 8 and 24 h, respectively (Fig. 1C and D).

Radiation impact on compactness. The compactness of oral mucosa models based on cell density and integrity was quantified from HE-stained cultures using at least 3 images of the same 3D culture. Radiation-induced loss of compactness was clearly observed, as shown in Fig. 2A. Computer-based analyses revealed that both heavy-ion irradiation with ${ }^{12} \mathrm{C}$ particles and $\mathrm{X}$-rays caused a significant loss of compactness in almost all irradiated samples (Fig. 2B and C). Co-cultures containing immune cells exhibited no clear tendency of changes in compactness, with the earliest effects occurring $24 \mathrm{~h}$ after irradiation with 2 Gy (Fig. 2D).

Radiation-induced DNA damage. Software-based evaluation of DSB was performed in representative images of $\gamma \mathrm{H} 2 \mathrm{AX}$ stainings. Controls showed barely visible DNA damage (Fig. 3A). In contrast, analyses of cultures $4 \mathrm{~h}$ after irradiation showed twice as many DSB after irradiation with heavy ions compared to X-rays (Fig. 3B and C). Both irradiation with heavy ions and with $\mathrm{X}$-rays showed a dose-dependent increase of DSB in all mucosa models, as shown in Fig. 3D and E. After an incubation time of $24 \mathrm{~h}$, the number of foci/nucleus in both radiation types aligned and decreased to the untreated level after 48 h. Samples, which were irradiated with 10 Gy of X-rays showed, after 24 and 48 h, significantly lower DSB levels compared to the corresponding dose after $4 \mathrm{~h}$. Similarly, $48 \mathrm{~h}$ after heavy ion irradiation with 4 or $10 \mathrm{~Gy}$, the quantity of DSB decreased significantly, compared to the corresponding doses after $4 \mathrm{~h}$.

Radiation-induced activation of $N F \kappa B$ p50. Fig. 4A-D show representative images of $\mathrm{NF} \kappa \mathrm{B}$ stainings on cryosections of 
A

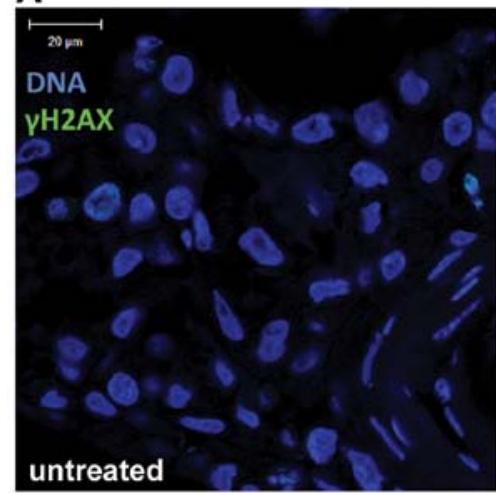

D

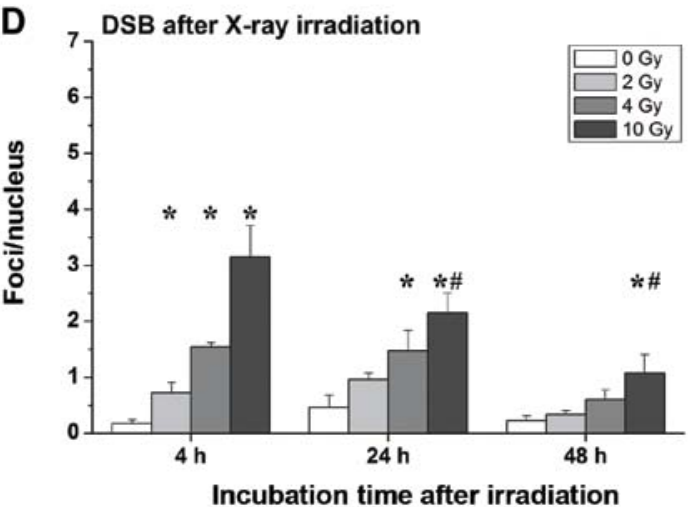

C
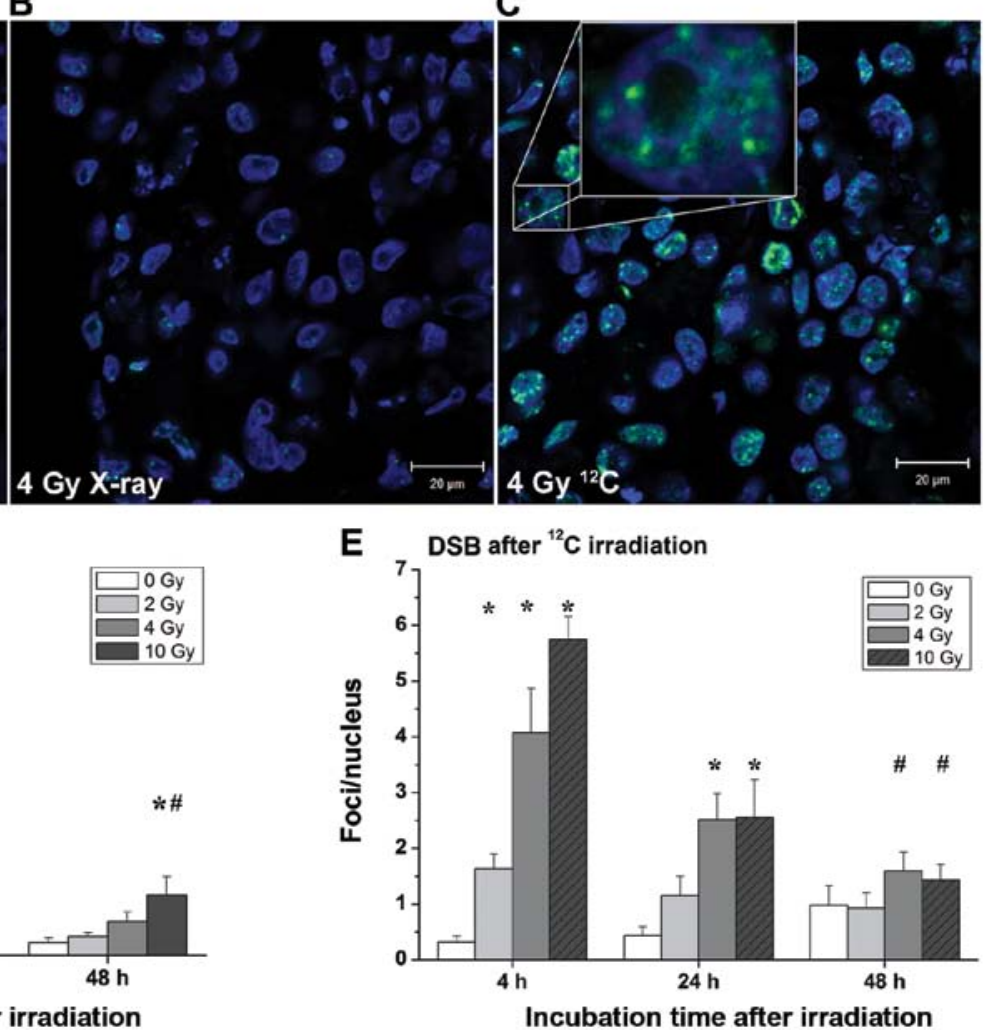

Figure 3. $\gamma \mathrm{H} 2 \mathrm{AX}$-stained DSB (green) in cryosections $4 \mathrm{~h}$ after irradiation with X-rays (B) and ${ }^{12} \mathrm{C}$ heavy ions (C). (A) Untreated control. DNA (blue) was stained with DAPI. The magnification in (C) shows an image detail of a cell nucleus with separate DSB as foci (green). Evaluation was carried out from at least 3 images/culture and 3 independent experiments. Values are shown in bar graphs (D and E) as means \pm SEM. ${ }^{*} \mathrm{p} \leq 0.05$ compared to untreated controls, ${ }^{\#} \mathrm{p} \leq 0.05$ compared to corresponding dose at $4 \mathrm{~h}$. Shaded bars in graph (E) denote results from only one experiment. DSB, double strand break.
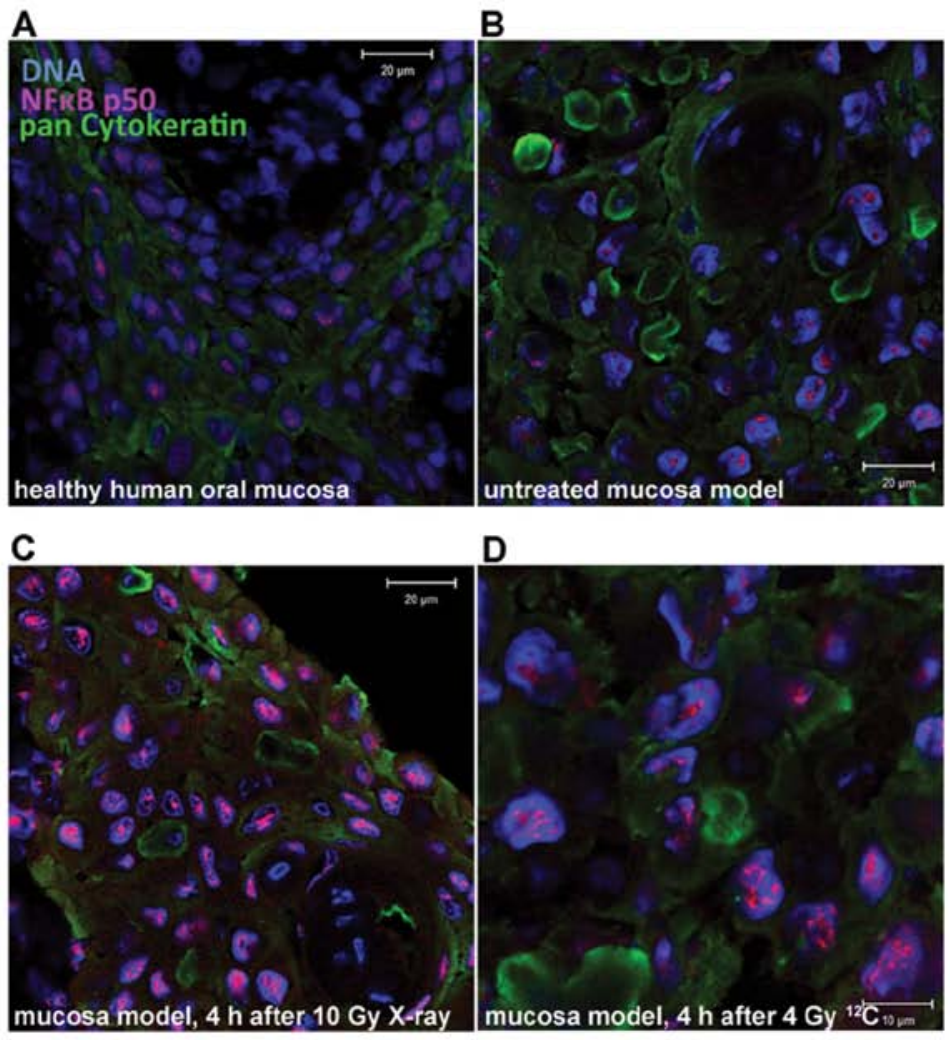

E $\quad$ NFKB p50 activation after X-ray irradiation

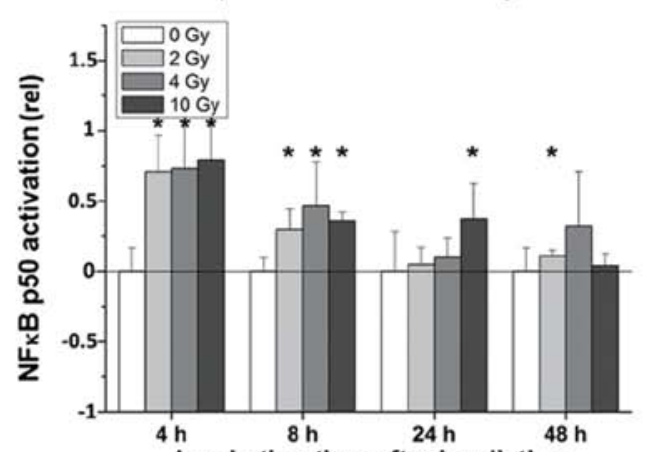

F NFKB p50 activation after ${ }^{12} \mathrm{C}$ irradiation

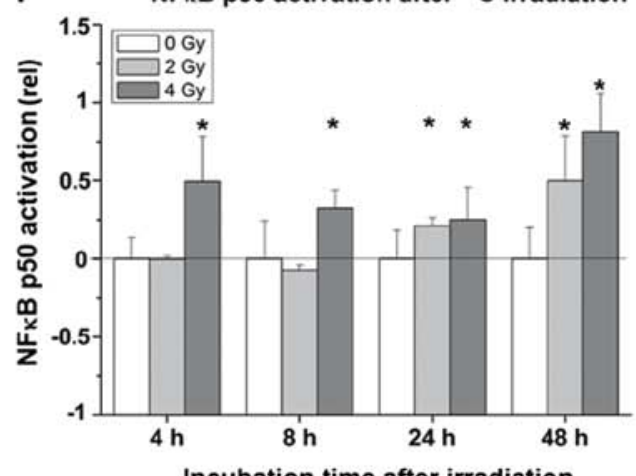

Figure 4. NFאB p50-stained cryosections of healthy human oral mucosa (A), controls (B), X-ray (C) and ${ }^{12} \mathrm{C}$ irradiated (D) mucosa models. Nuclear located NFKB p50 (pink) was evaluated from at least 3 images/cultures and 3 independent experiments. Values are shown in bar graphs (E and F) as means \pm SEM. $\mathrm{NF \kappa B}$ p50 activation is given in relative units. The background activation level was set to 0 . ${ }^{*} \mathrm{p} \leq 0.05$ compared to untreated controls. 


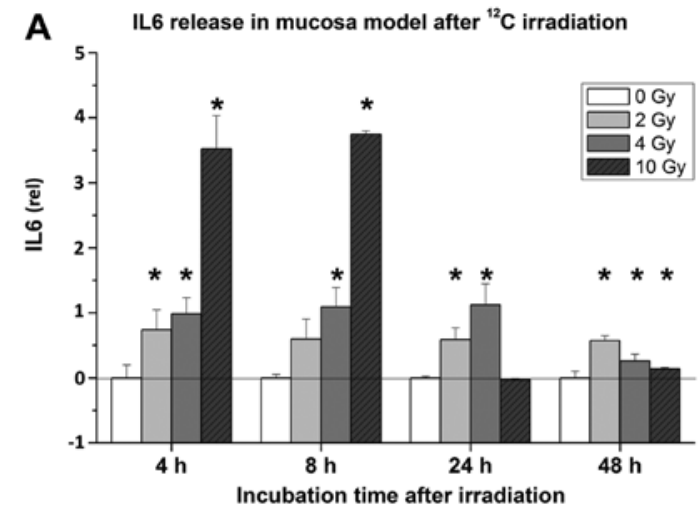

B

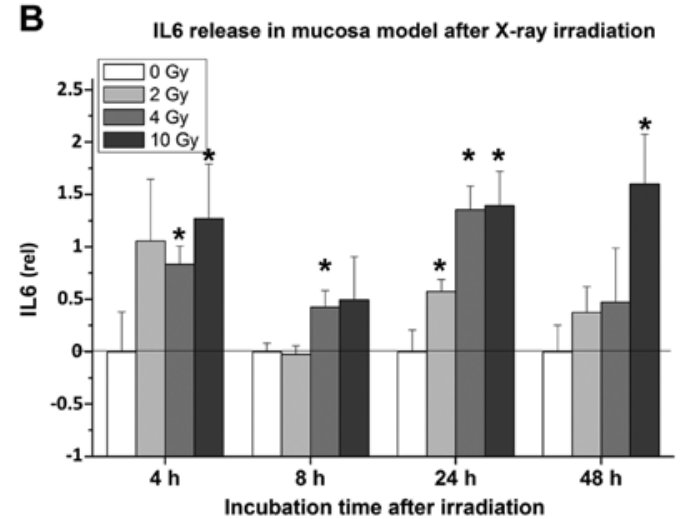

C

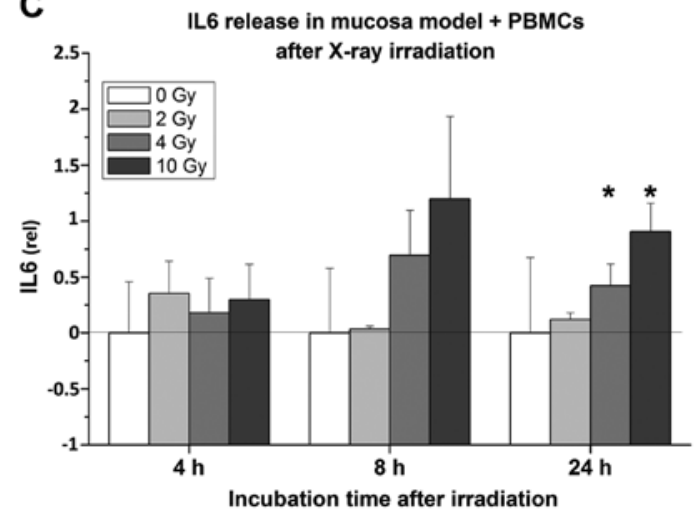

Figure 5. IL6 release in oral mucosa models measured after irradiation with ${ }^{12} \mathrm{C}$ heavy ions (A) and X-ray (B). Release profile of IL6 in mucosa models containing PBMCs (C) corresponds roughly to cultures without immune cells. IL6 release is given in relative units and normalized to untreated controls, which were set to 0 . ${ }^{*} \mathrm{p} \leq 0.05$ compared to untreated controls. Shaded bars in graph (A) denote results from only one experiment. IL6, interleukin 6; PBMCs, peripheral blood mononuclear cells.

the mucosa model. A clear accumulation of the transcription factor in the nucleus according to its activation pattern was observed after exposure to both types of radiation in all mucosa models (Fig. 4C and D). Software-based evaluations confirmed these observations (Fig. 4E and F). X-rays induced $\mathrm{NF \kappa B}$ p50 activation almost immediately after irradiation, with statistically significant changes at all doses after 4 and $8 \mathrm{~h}$. The NFkB p50 activation decreased after 24 and $48 \mathrm{~h}$ to the untreated level, significant changes were detected only after exposure to $10 \mathrm{~Gy}$ after $24 \mathrm{~h}$ and to $2 \mathrm{~Gy}$ after $48 \mathrm{~h}$. On the other hand, ${ }^{12} \mathrm{C}$ particles induced significant changes in $\mathrm{NF \kappa B}$ activation $24 \mathrm{~h}$ after $2 \mathrm{~Gy}$ and $48 \mathrm{~h}$ after $4 \mathrm{~Gy}$.
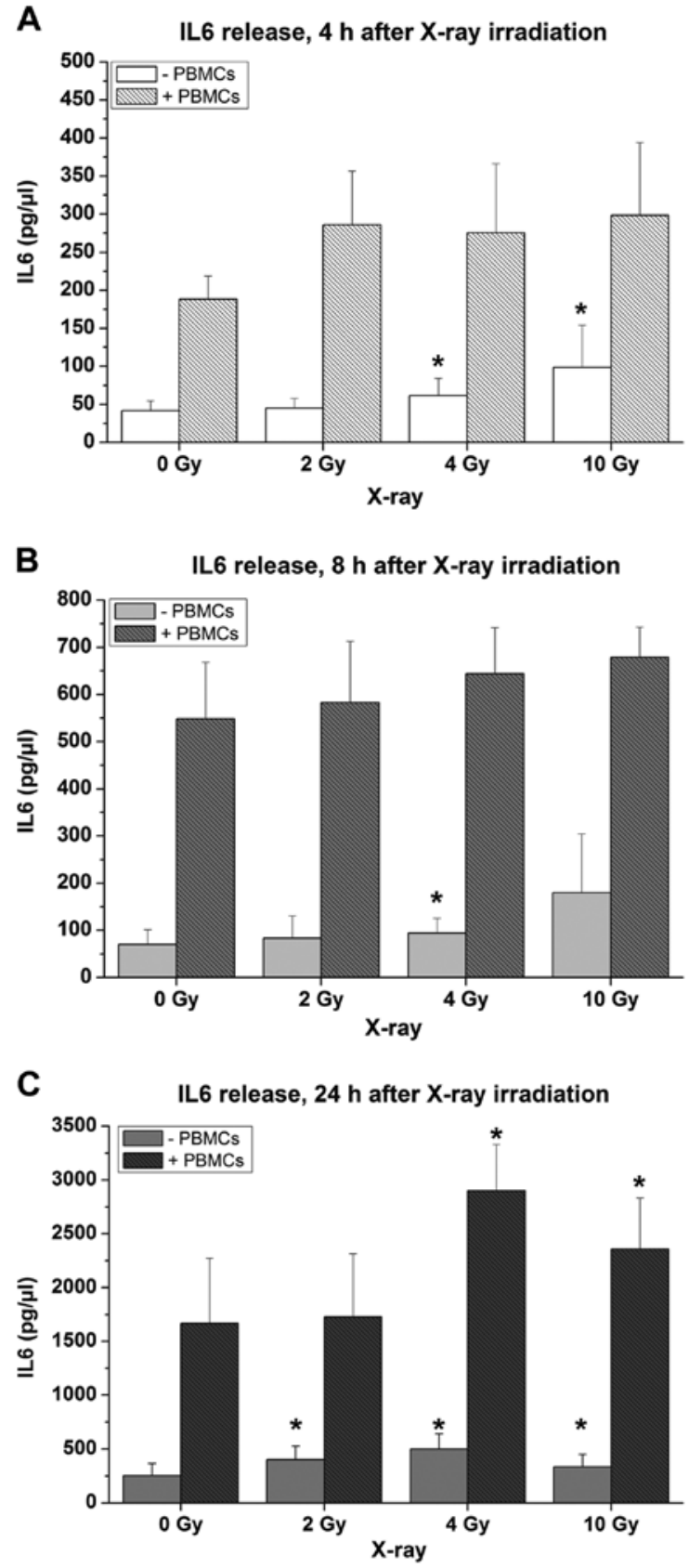

Figure 6 . Absolute IL6 concentrations (pg/ $\mu 1$ ) released by mucosa models with and without PBMCs after X-ray irradiation. Immune cells induced 2-3-fold higher cytokine concentrations $4 \mathrm{~h} \mathrm{(A),} 8 \mathrm{~h}$ (B) and $24 \mathrm{~h}$ (C) after irradiation, respectively. IL6, interleukin 6; PBMCs, peripheral blood mononuclear cells.

Radiation impact on IL6 and IL8 release. The 3D organotypic mucosa models immediately responded to radiation of both qualities by secretion of cytokines and chemokines, but there were still several differences in cytokine release. Irradiation with the particle beam caused the strongest effect after 4 and $8 \mathrm{~h}$, while $24 \mathrm{~h}$ after exposure to $10 \mathrm{~Gy}$, the levels of IL6 decreased, as depicted in Fig. 5A. The contrary effect was observed in mucosa models exposed to X-rays, where the levels of IL6 increased over time and reached the maximum $24 \mathrm{~h}$ after treatment (Fig. 5B). Co-cultures containing immune cells revealed similar responses to $\mathrm{X}$-ray treatment, but significant increase of IL6 levels were detected only after $24 \mathrm{~h}$ (Fig. 5C). 

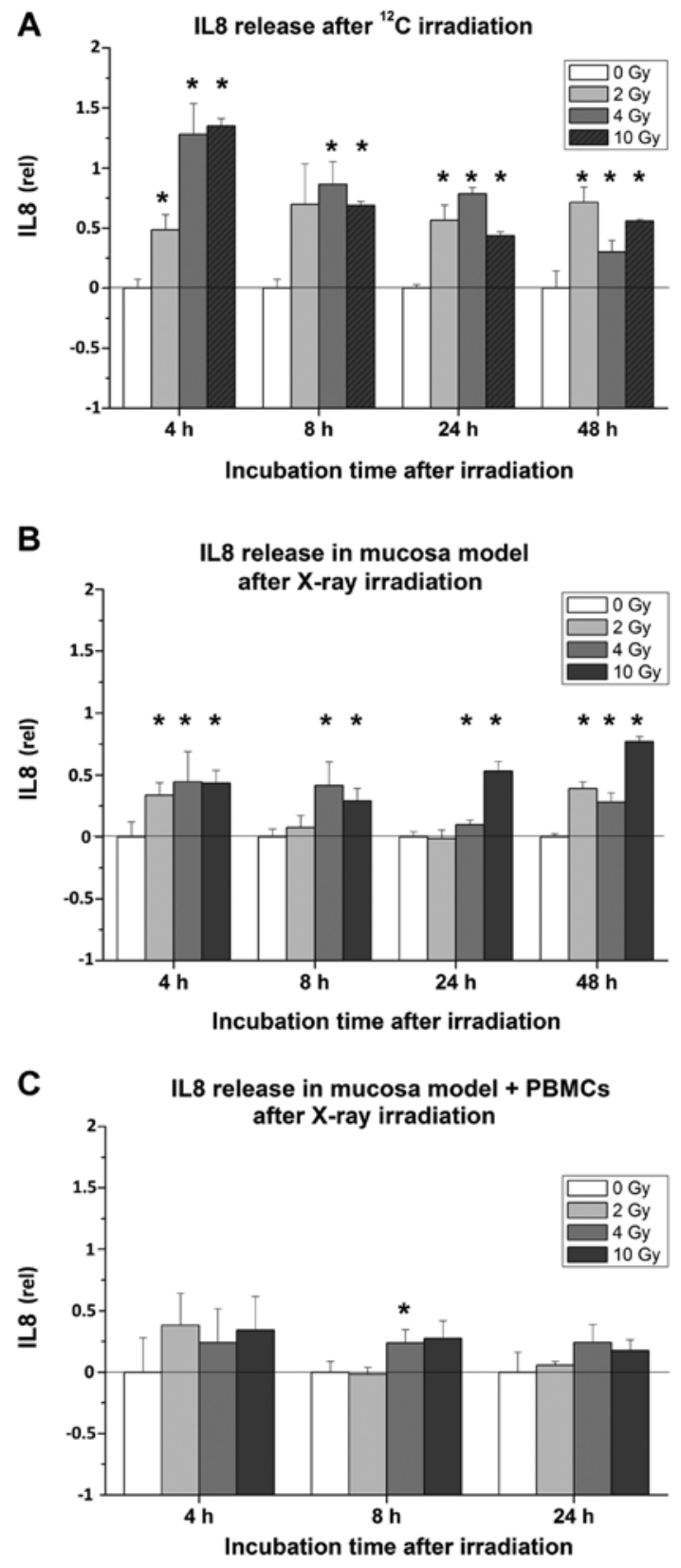

Figure 7. IL8 release in oral mucosa models measured after irradiation with ${ }^{12} \mathrm{C}$ heavy ions (A) and X-rays (B). IL8 release in mucosa models containing PBMCs (C) showed a tendency to increase with significant more chemokine $24 \mathrm{~h}$ after irradiation with 2 Gy. IL8 release is given in relative units and normalized to untreated controls, which were set to 0 . $p \leq 0.05$ compared to untreated controls. Shaded bars in graph (A) denote results from only one experiment. IL8, interleukin 8; PBMCs, peripheral blood mononuclear cells.
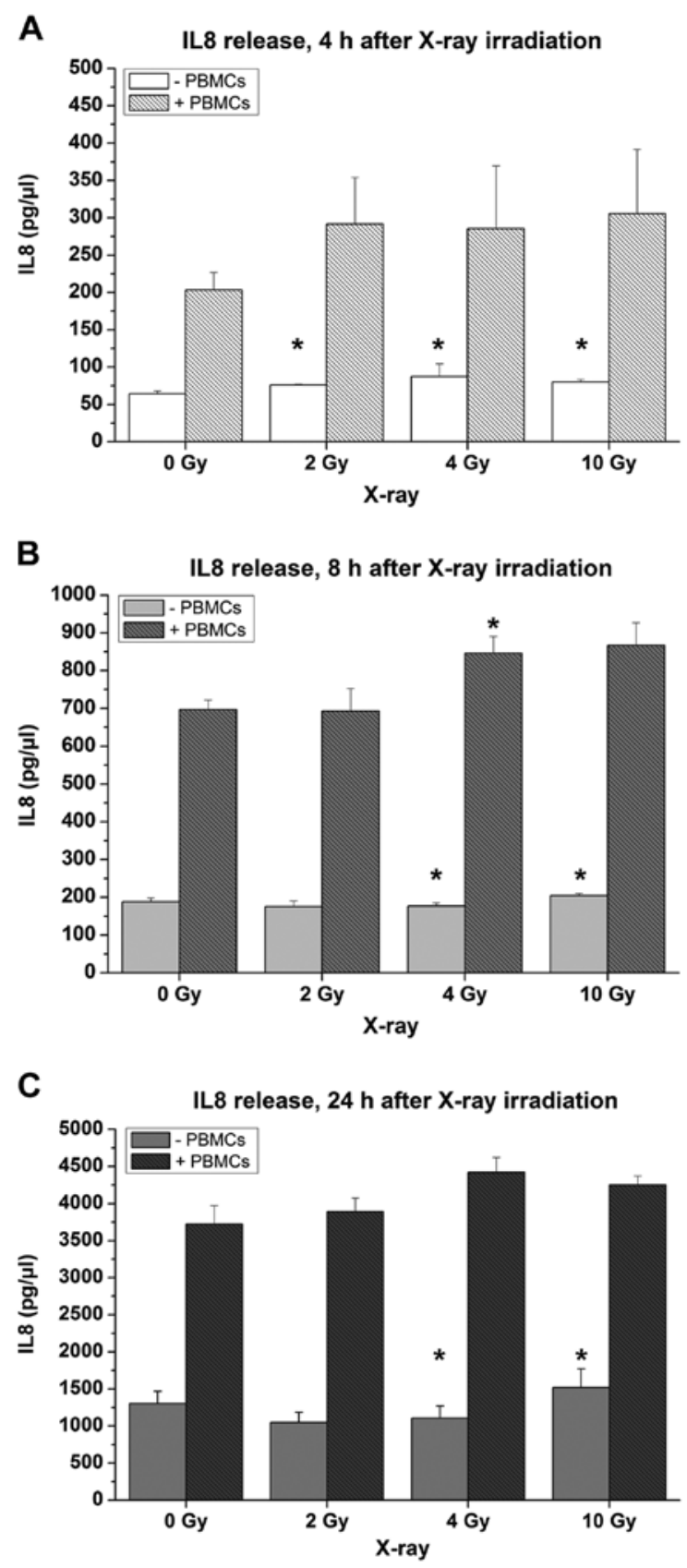

Figure 8 . Absolute IL8 concentrations (pg/ $\mu 1$ ) released by mucosa models with and without PBMCs after X-ray irradiation. Immune cells induced 2-3-fold higher cytokine concentrations $4 \mathrm{~h}$ (A), $8 \mathrm{~h}$ (B) and $24 \mathrm{~h}$ (C) after irradiation, respectively. IL8, interleukin 8; PBMCs, peripheral blood mononuclear cells.
Here, 2-3-fold higher IL6 concentrations compared to mucosa models without PBMCs were observed (Fig. 6).

After irradiation with ${ }^{12} \mathrm{C}$ particles, organotypic cultures consistently released significantly more IL8, compared to untreated samples, with the maximum after $4 \mathrm{~h}$ (Fig. 7A). Subsequently, the levels of IL8 decreased gradually over time. Mucosa models treated with $\mathrm{X}$-rays showed the strongest effects at a dose of $10 \mathrm{~Gy}$ and $48 \mathrm{~h}$ after irradiation (Fig. 7B). Co-cultures including PBMCs revealed a tendency to a higher release of IL8 after X-ray irradiation compared to untreated samples (Fig. 7C) and 2-3-fold higher IL8 concentrations compared to mucosa models without PBMCs (Fig. 8).

\section{Discussion}

Although X-ray and heavy-ion radiotherapy have been applied in cancer treatment, several adverse events restrict the therapeutic success. Radiation-induced oral mucositis is a well-documented painful side-effect of radiation therapy $(6,7,9,11)$; however, our understanding of the underlying cellular mechanisms is limited. The present study investigated the radiation impact on oral mucosa, using a 3D organotypic culture with and without human immune cells, comparing X-rays and ${ }^{12} \mathrm{C}$ heavy-ion irradiation. In addition to the clinical aspects, the present study assessed the risk of 
developing radiation-induced oral mucositis during extended space flights.

Previous studies revealed increased cytokeratin expression in the initial stage of oral mucositis in biopsies of irradiated patients linked to the proliferation of epithelium cells during the initial phases of radiotherapy (23). Biopsies were obtained from buccal mucosa, when patients had already received a radiation dose of $36 \mathrm{~Gy}$ and had presented mucositis grade 1 . In the present study, however, irradiation of organotypic cultures with ${ }^{12} \mathrm{C}$ particles at a dose up to $4 \mathrm{~Gy}$ showed no effects on the proportion of the epithelium within the $3 \mathrm{D}$ cultures, whereas X-ray irradiation with up to $10 \mathrm{~Gy}$ appeared to reduce the epithelium. Furthermore, ${ }^{12} \mathrm{C}$ ion irradiation caused a more pronounced loss of compactness within the mucosa model cultures than $\mathrm{X}$-ray irradiation. These differences in the effect of ${ }^{12} \mathrm{C}$ ions and $\mathrm{X}$-rays most likely result from the different physical qualities of these radiation types.

It was also important to analyze the radiation-induced DNA damage, since previous studies showed that DSB may activate NFKB and the following signaling pathway by mediator molecules such as ATM or DNA-dependent protein kinase (17,24-26). Analyses of radiation-induced DSB in our mucosa model revealed a clear dose-dependent increase of $\gamma \mathrm{H} 2 \mathrm{AX}$ foci $4 \mathrm{~h}$ after irradiation with carbon ions and X-rays. As a result of this DNA damage, the foundation for induction of inflammatory response, such as activation of $\mathrm{NF \kappa B}$ p50 and the release of IL6 and IL8, was laid. Subsequently, $48 \mathrm{~h}$ after irradiation, the DSB decreased to the untreated level, although the incidence and severity of oral mucositis is not necessarily correlated with DSB repair (10).

Activation of the transcription factor $\mathrm{NF} \kappa \mathrm{B}$ and increased secretion of pro-inflammatory cytokines and chemokines are involved not only in initiation of radiation-induced oral mucositis $(22,27)$, but also in linking inflammation to cancer development and progression (14-17,28). We observed carbon ion-induced activation of $\mathrm{NF \kappa B}$ in the mucosa model, which has been already documented for HEK cells (29). Cultures treated with X-rays showed a distinct NFкB p50 translocation to the nucleus as well. Again, the different qualities of radiation appear to affect mucosa cultures in different ways following different kinetics. X-rays induced an early activation of $\mathrm{NF \kappa B}$ already $4 \mathrm{~h}$ after treatment, which returned to control levels at $24 \mathrm{~h}$ after treatment. This is in contrast to heavy-ion-induced effects, which reached their maximum $48 \mathrm{~h}$ after treatment.

Similar to other studies (30-32), we observed an increased expression of IL6 and IL8 as a probable response to preceding NFкB p50 activation. Notably, the analyses of IL6 signal revealed a reverse behavior, compared to $\mathrm{NF \kappa B}$ p50 activation. In the present study, carbon ion irradiation induced the strongest effects 4 and $8 \mathrm{~h}$ after radiation, response to X-rays was the highest 24 and $48 \mathrm{~h}$ after treatment. The kinetics of $\mathrm{NF} \kappa \mathrm{B}$ activation and the IL6 release appear to occur with a time delay. This may indicate a causative sequence of events. The ${ }^{12} \mathrm{C}$-induced segregation of the chemokine IL8 follows a similar release profile as IL6. In contrast to IL6, the signal for IL8 induced by X-rays reached its maximum at $8 \mathrm{~h}$ and decreased $24 \mathrm{~h}$ after treatment. Co-cultures containing PBMCs responded qualitatively similar to mucosa models without immune cells, but showed much higher concentrations for IL6 and IL8, respectively, which makes the mucosa model more sensitive for radiation-induced responses. Since a correlation between increased expression of IL8 and carcinogenesis for several entities has been previously described (15,16,33-35), our results may indicate an increased risk of tumorigenesis for astronauts during prolonged space missions.

In conclusion, reproducible inflammatory responses induced by sparsely and densely ionizing radiation were detected as a sign of the initiation stage of oral mucositis in $3 \mathrm{D}$ organotypic mucosa models with and without immune cells. Furthermore, first precursors of carcinogenesis were determined. Nevertheless, an assessment of the risk of developing radiation-induced oral mucositis during prolonged space flights proves to be rather difficult, since the induction of inflammatory processes depends on various determinants, such as the quality of the spacecraft shielding or accumulation dose. To avoid these complications and to ensure safety for crew members, ESA and other space agencies are working on evaluating possible improvements in radiation shielding approaches (3). For the consistent reduction of risk, a cautious management and convenient timing for such prolonged missions is required.

\section{Acknowledgements}

The present study was supported by the European Space Agency (ESA), the German Aerospace Center (DLR), the Federal Ministry of Economics and Technology (BMWi, no. 50 WB 0926), and the GSI Helmholtz Center for Heavy Ion Research in Darmstadt. The authors thank the laboratory of Dr Susanne Strand, University Medical Center Mainz, particularly Dr Steffen Lorenz, for LSM imaging and the GSI Biophysics accelerator team for the support during beam times.

\section{References}

1. Hall EJ and Giaccia A: Radiobiology for the Radiologist. 7th edition. Lippincott Williams \& Wilkins, Philadelphia, PA, 2012.

2. Hawkey A: Physiological and biomechanical considerations for a human Mars mission. J Br Interplanet Soc 58: 117-130, 2005.

3. Cucinotta FA, Kim MHY and Chappell LJ: Evaluating shielding approaches to reduce space radiation cancer risks. NASA Center for AeroSpace Information May: 1-35, 2012.

4. Cucinotta FA, Kim MHY and Ren L: Evaluating shielding effectiveness for reducing space radiation cancer risks. Radiat Meas 41: 1173-1185, 2006.

5. Durante $M$ and Cucinotta FA: Heavy ion carcinogenesis and human space exploration. Nat Rev Cancer 8: 465-472, 2008.

6. Schulz-Ertner D, Haberer T, Scholz M, et al: Acute radiationinduced toxicity of heavy ion radiotherapy delivered with intensity modulated pencil beam scanning in patients with base of skull tumors. Radiother Oncol 64: 189-195, 2002.

7. Sonis ST and Fey EG: Oral complications of cancer therapy. Oncology 16: 680-686, 2002.

8. Vissink A, Jansma J, Spijkervet FK, Burlage FR and Coppes RP: Oral sequelae of head and neck radiotherapy. Crit Rev Oral Biol Med 14: 199-212, 2003.

9. Jensen AD, Nikoghosyan AV, Ecker S, Ellerbrock M, Debus J and Münter MW: Carbon ion therapy for advanced sinonasal malignancies: feasibility and acute toxicity. Radiat Oncol 6: 30, 2011.

10. Fleckenstein J, Kühne M, Seegmüller K, et al: The impact of individual in vivo repair of DNA double-strand breaks on oral mucositis in adjuvant radiotherapy of head-and-neck cancer. Int J Radiat Oncol Biol Phys 81: 1465-1472, 2011. 
11. Sonis ST: Oral mucositis in head and neck cancer: risk, biology, and management. Am Soc Clin Oncol Educ Book, pp236-240, 2013.

12. Naidu MU, Ramana GV, Rani PU, Mohan IK, Suman A and Roy P: Chemotherapy-induced and/or radiation therapyinduced oral mucositis - complicating the treatment of cancer. Neoplasia 6: 423-431, 2004.

13. Hickok JT, Morrow GR, Roscoe JA, Mustian K and Okunieff P: Occurrence, severity, and longitudinal course of twelve common symptoms in 1129 consecutive patients during radiotherapy for cancer. J Pain Symptom Manage 30: 433-442, 2005.

14. Porta C, Larghi P, Rimoldi M, Totaro MG, Allavena P, Mantovani A and Sica A: Cellular and molecular pathways linking inflammation and cancer. Immunobiology 214: 761-777, 2009.

15. Karin $\mathrm{M}$ and Greten FR: NF- $\mathrm{BB}$ : linking inflammation and immunity to cancer development and progression. Nat Rev Immunol 5: 749-759, 2005.

16. Karin M: NF- $\kappa B$ as a critical link between inflammation and cancer. Cold Spring Harb Perspect Biol 1: a000141, 2009.

17. Magné N, Toillon RA, Bottero V, Didelot C, Houtte PV, Gérard JP and Peyron JF: NF- $\kappa \mathrm{B}$ modulation and ionizing radiation: mechanisms and future directions for cancer treatment. Cancer Lett 231: 158-168, 2006.

18. Boxman IL, Ruwhof C, Boerman OC, Löwik CW and Ponec M: Role of fibroblasts in the regulation of proinflammatory interleukin IL-1, IL-6 and IL-8 levels induced by keratinocyte-derived IL-1. Arch Dermatol Res 288: 391-398, 1996.

19. Sawicki G, Marcoux Y, Sarkhosh K, Tredget E and Ghahary A: Interaction of keratinocytes and fibroblasts modulates the expression of matrix metalloproteinases-2 and -9 and their inhibitors. Mol Cell Biochem 269: 209-216, 2005.

20. Wang Z, Wang Y, Farhangfar F, Zimmer $M$ and Zhang $Y$ Enhanced keratinocyte proliferation and migration in co-culture with fibroblasts. PLoS One 7: e40951, 2012.

21. Werner S, Krieg T and Smola H: Keratinocyte-fibroblast interactions in wound healing. J Invest Dermatol 127: 998-1008, 2007.

22. Sonis ST: The pathobiology of mucositis. Nat Rev Cancer 4: $277-284,2004$
23. Bonan PRF, Kaminagakura E, Pires FR, Vargas PA and de Almeida OP: Cytokeratin expression in initial oral mucositis of head and neck irradiated patients. Oral Surg Oral Med Oral Pathol Oral Radiol Endod 101: 205-211, 2006.

24. Basu S, Rosenzweig KR, Youmell M and Price BD: The DNA-dependent protein kinase participates in the activation of NFאB following DNA damage. Biochem Biophys Res Commun 247: 79-83, 1998.

25. Lee SJ, Dimtchev A, Lavin MF, Dritschilo A and Jung M: A novel ionizing radiation-induced signaling pathway that activates the transcription factor NF- $\kappa$ B. Oncogene 17: 1821-1826, 1998.

26. Piret B, Schoonbroodt S and Piette J: The ATM protein is required for sustained activation of NF- $\kappa$ B following DNA damage. Oncogene 18: 2261-2271, 1999.

27. Sonis ST: New thoughts on the initiation of mucositis. Oral Dis 16: 597-600, 2010.

28. Ben-Neriah Y and Karin M: Inflammation meets cancer, with $\mathrm{NF}-\kappa \mathrm{B}$ as the matchmaker. Nat Immunol 12: 715-723, 2011.

29. Hellweg CE, Baumstark-Khan C, Schmitz C, et al: Carbonion-induced activation of the NF- $\mathrm{KB}$ pathway. Radiat Res 175: 424-431, 2011.

30. Colley HE, Eves PC, Pinnock A, Thornhill MH and Murdoch C: Tissue-engineered oral mucosa to study radiotherapy-induced oral mucositis. Int J Radiat Biol 89: 907-914, 2013.

31. Tobita T, Izumi K and Feinberg SE: Development of an in vitro model for radiation-induced effects on oral keratinocytes. Int J Oral Maxillofac Surg 39: 364-370, 2010.

32. Lambros MP, Parsa C, Mulamalla $\mathrm{H}$, et al: Identifying cell and molecular stress after radiation in a three-dimensional (3-D) model of oral mucositis. Biochem Biophys Res Commun 405: 102-106, 2011.

33. Brat DJ, Bellail AC and Van Meir EG: The role of interleukin-8 and its receptors in gliomagenesis and tumoral angiogenesis. Neuro Oncol 7: 122-133, 2005

34. Xie K: Interleukin-8 and human cancer biology. Cytokine Growth Factor Rev 12: 375-391, 2001.

35. Yin Y, Si X, Gao Y, Gao L and Wang J: The nuclear factor- $\kappa \mathrm{B}$ correlates with increased expression of interleukin- 6 and promotes progression of gastric carcinoma. Oncol Rep 29: 34-38, 2013. 